\title{
Catarata em corticoterapia sistêmica: prevalência e relação com tempo e dose cumulativa de glicocorticóides
}

\author{
Corticosteroid therapy and cataract:prevalence and relation to timeof use and cumu- \\ lativedose
}

\author{
Fernanda Ziger ${ }^{1}$ \\ Rommel Josuel Zago ${ }^{2}$ \\ Marcelo Gehlen ${ }^{3}$ \\ Marília B. Silva ${ }^{4}$ \\ Thelma L. Skare ${ }^{4}$
}

Serviços de Oftalmologia e Reumatologia do Hospital Evangélico Universitário de Curitiba, Paraná, Brasil ${ }^{1}$ R2 de Clínica Médica do Hospital Universitário Evangélico de Curitiba

${ }^{2}$ R2 de Oftalmologia do Hospital Universitário Evangélico de Curitiba

3 Professor Auxiliar da Disciplina de Oftalmologia da Faculdade Evangélica de Medicina do Paraná

Professora Assistente da Disciplina de Reumatologia da Faculdade Evangélica de Medicina do Paraná

Endereço para correspondência: Alameda Princesa Izabel, 1464 - ap. 84 - Curitiba (PR) CEP 80730-080 E-mail:-smgehlen@terra.com.br

Recebido para publicação em 19.11.2001 Aceito para publicação em 11.09.2002

Nota Editorial: Pela análise deste trabalho e por sua anuência na divulgação desta nota, agradecemos ao Dr. Leonardo Akaishi.

\begin{tabular}{l} 
RESUMO \\
\hline Objetivo: O presente estudo teve por finalidade estudar a freqüência de \\
catarata em pacientes reumáticos e usuários crônicos de corticóide, \\
procurando-se correlacionar o seu aparecimento com tempo de uso, dose \\
cumulativa total e doença de fundo. Métodos: Foram estudados 27 \\
pacientes reumáticos usuários crônicos de corticóide, com exame de \\
lâmpada de fenda e calculado o tempo de uso e doses cumulativas de \\
prednisona ou equivalente. Resultado: Encontrou-se freqüência de \\
$18,52 \%$ de cataratas e não foi possível demonstrar correlação com dose \\
cumulativa do medicamento (p=0,231) ou com o tipo de doença de fundo. \\
Apesar do tempo médio de uso de corticóide ter sido maior entre os \\
pacientes que apresentaram catarata, esta correlação não demostrou \\
significância estatística (p=0,694). Conclusão: Os autores concluem que \\
esta complicação érelativamente comum e que mais estudos são necessá- \\
rios para melhor entender o processo fisiopatológico implicado na sua \\
formação.
\end{tabular}

Descritores: Catarata/fisiopatologia; Catarata/etiologia; Doenças reumáticas/quimioterapia; Artrite reumatóide/quimioterapia; Corticosteróides/efeitos adversos; Corticoisteróides/ uso terapêutico; Tolerância a drogas

\section{INTRODUĈ̃̃O}

Desde a sua descoberta na década de 1940 por P. Hench ${ }^{(1)}$, os glicocorticóides são drogas amplamente utilizadas para tratamento de uma grande variedade de doenças devido às suas propriedades antiinflamatórias e imunossupressoras. Embora o uso deste medicamento tenha modificado a evolução natural de muitas doenças, algumas das quais potencialmente fatais, é patente a vasta gama de seus efeitos colaterais que em muito cerceia o seu uso.

Alguns dos efeitos colaterais dos glicocorticóides são amplamente estudados e graças ao reconhecimento das nuances que levam ao seu aparecimento podem ser prevenidos ou minimizados, como é o caso, por exemplo, da osteoporose. Outros já são bem menos conhecidos como acontece com manifestações oculares. No entanto, é mister que também esta última forma de efeitos colaterais seja reconhecida e adequadamente valorizada para que, em situações de uso crônico dos glicocorticóides, o seu impacto possa ser diminuído.

Este trabalho estuda a possível correlação entre o uso de glicocorticóides com o aparecimento de cataratas subcapsulares posteriores em usuários crônicos deste medicamento. 


\section{MÉTODOS}

O estudo foi aprovado pelo Comitê de Ética em Pesquisa, ligado ao Instituto de Pesquisas do Hospital Universitário Evangélico de Curitiba, PR.

Foram estudados 27 pacientes reumáticos, usuários crônicos de glicocorticóide por via oral, com idade entre 15 e 68 anos (média: 38,56 $\pm 13,02$ ), sendo 26 mulheres e 1 homem. O estudo foi realizado no período de junho a agosto de 2001 nos ambulatórios de Reumatologia e de Oftalmologia do Hospital Universitário Evangélico de Curitiba.

As doenças que levaram à indicação de uso deste medicamento eram lúpus eritematoso sistêmico ( LES ) em 20 deles; lúpus discóide (LED) em 1; artrite reumatóide (AR) em 3; Síndrome de Sjögren (SS) em 1; e outras vasculites em 2. O tempo de duração da doença variou entre 1 e 21 anos e o tempo de uso do glicocorticóide ficou entre 8 e 240 meses. A dose cumulativa utilizada desta droga variou entre $2,18 \mathrm{~g}$ e $62,53 \mathrm{~g}$ de prednisona ou equivalente (média de 14,63g \pm $15,34 \mathrm{~g})$.

O diagnóstico de catarata foi feito pelo exame oftalmológico com lâmpada de fenda; os pacientes foram avaliados sempre pelo mesmo examinador. Excluíram-se doenças retinianas e outras alterações oculares que pudessem diminuir a acuidade visual ou causar catarata secundária através do exame de oftalmoscopia indireta.

Os dados obtidos foram estudados por tabelas de freqüência e de contingência e pelos testes Exato de Fisher e MannWhitney. Nível de significância adotado de 5\%.

\section{RESULTADOS}

Dos 27 pacientes estudados, 6 (ou 18,52\%) apresentaram evidências de catarata.

Estudando-se a relação entre o tempo de doença e a presença de catarata verificou-se não existir correlação entre estas duas variáveis (Exato de Fisher; $\mathrm{p}=0,6122$ ).

Apesar do tempo médio de uso de corticóide ter sido maior entre os pacientes que apresentaram catarata, não existiram evidências suficientes para afirmar que o tempo de uso causa maior freqüência de catarata, pelo Teste de Mann-Whitney $(\mathrm{p}=0,694)($ tabela 1$)$.

Estudando-se a correlação entre dose cumulativa total ingerida de corticóide e a presença de catarata observou-se que não existem evidências para afirmar que existe correlação entre

\begin{tabular}{|cc|}
\hline \multicolumn{2}{|c|}{$\begin{array}{c}\text { Tabela 1. Catarata e tempo de uso de corticoide } \\
\text { Catarata e tempo médio de uso de corticóide }\end{array}$} \\
\begin{tabular}{|cc} 
Pacientes & Tempo médio de uso de corticóide \\
(tempo em meses \pm desvio padrão)
\end{tabular} \\
$\begin{array}{lc}\text { Com catarata }(n=5) & 63,00 \pm 99,52 \\
\text { Sem catarata }(n=22) & 33,59 \pm 23,44 \\
n=\text { número de pacientes da amostra estudada; } p=0,694\end{array}$ \\
\hline
\end{tabular}

estas duas variáveis (Mann Whitney; $\mathrm{p}=0,231$ ) como pode ser observado no gráfico 1 .

Por último, procurou-se estudar se a presença de catarata estava relacionada com o diagnóstico de LES, também não se encontrando correlação ( Exato de Fisher; $p=0,6122$ ).

\section{DISCUSSÃO}

O estudo dos efeitos colaterais dos glicocorticóides mostra que estes podem ser divididos em dois grandes grupos: os precoces, como acne, alterações no humor, ganho de peso etc, (que se revertem ou minimizam assim que a droga é retirada) e os tardios como osteoporose, catarata e necrose avascular (os quais causam dano permanente, irreversível) ${ }^{(2)}$.

Apesar do aparecimento da catarata com uso do corticóide ser amplamente reconhecido, o mecanismo pelo qual isto acontece não está elucidado e nem tem recebido dos pesquisadores a importância devida ${ }^{(2)}$. Sabe-se apenas que, assim como a osteoporose, esta é uma complicação que não é prevenida pelo uso de corticosteróides em dias alternados ${ }^{(2)}$.

Os pacientes aqui estudados mostraram uma alta freqüência de cataratas (em torno de $18,52 \%$ ) o que aponta para o fato de que esta é uma complicação relativamente comum. Estudos na literatura feitos unicamente em pacientes com LES apontam para freqüência de $10 \%$ mas, por terem sido feitos em população exclusiva de lúpicos, não permitiram separar os efeitos dos glicocorticóides daquelas da doença de base ${ }^{(3)}$. No estudo atual, a doença reumática de fundo que levou ao uso do corticóide pareceu não influir no aparecimento da catarata, pelo menos no casos dos portadores de LES, que eram a maioria dos pacientes estudados.

Alguns autores sugerem que tanto a dose cumulativa total como o tempo de uso são os elementos determinantes do aparecimento desta complicação. Isto não pode ser demonstrado neste pequeno estudo. Aliás, aqui existiu uma tendência para que o tempo de uso do medicamento fosse o responsável, embora não se tenha podido demonstrar uma significância

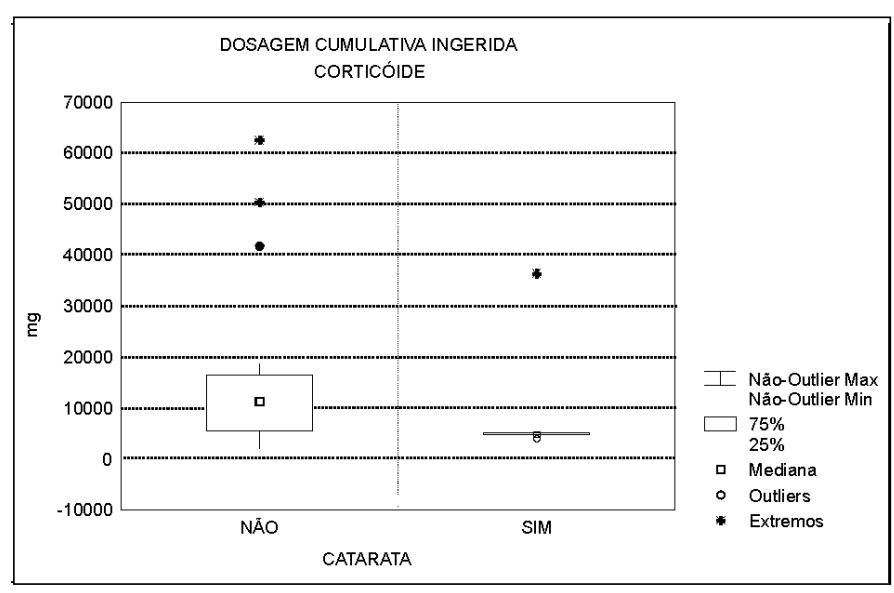

Figura 1 - Relação entre dose cumulativa total de corticosteróide (prednisona) e incidência de catarata 
estatística. Este estudo possui uma amostra pequena sendo difícil se calcular a dose cumulativa nestes pacientes de maneira fidedigna.

\section{CONCLUSÃO}

São necessários outros estudos para esclarecer aspectos da fisiopatologia desta complicação, um vez que este medicamento é amplamente utilizado não só em medicina interna, mas, também, na própria oftalmologia onde pacientes com problemas oculares ao serem tratados com corticóides vêem o problema da catarata se somar aos já existentes.

\section{ABSTRACT}

Purpose: To study cataract prevalence in rheumatic patients who used corticosteroid chronically and to correlate it with time and total corticosteroid dose as well as with the disease that required such treatment. Methods: We studied 27 rheumatic patients regarding cataract using slit lamp and calculated the total dose of prednisone or equivalent steroids as well as the treatment time. Results: We found an $18.52 \%$ prevalence and no correlation with cumulative dose $(p=0.231)$ or with the underlying disease. Although the time of treatment was longer in the group with cataract, no statistically significant difference could be found $(\mathrm{p}=0.694)$. Conclusion: The authors conclude that cataract is a relativelly common complication of steroid use and that further studies are needed to understand the pathophysiologic process of its formation.

Keywords: Cataract/physiopathology;Cataract/etiology; Rheumatic diseases/drug therapy; Rheumatoid arthritis/drug therapy; Adrenal cortex hormones/therapeutic use; Adrenal cortex hormones/adverse effects; Drug tolerance

\section{REFERÊNCIAS}

1. Chrousus GP. The hypothalamic-pituitary-adrenal axis and immune mediated inflammation [commented on N Engl J Med 1995;333:942-3]. N. Engl J Med 1995;332:1351-62.

2. Lester RE, Knowles SR, Shear NH. The risks of systemic corticosteroid use. Dermatol Clin 1998;16:277-88.

3. Zonana- Nacach A Barr SG, Laurence L, Petri M. Damage in systemic lupus erythematosus and its association with corticosteroids. Arthritis Rheum 2000;43:1801-8.

\title{
II Congresso Nacional da SBO Controvérsias e Casos Desafiadores
}

\author{
19 a 21 de Junho de 2003
}

Centro de Convenções Ribalta - Rio de Janeiro - RJ

\section{Promoção:}

Sociedade Brasileira de Oftalmologia (SBO) 